\title{
Role of magnetic chirality in polarization flip upon commensurate-incommensurate magnetic phase transition in $\mathrm{YMn}_{2} \mathrm{O}_{5}$
}

\author{
Shuichi Wakimoto, ${ }^{1}$ Hiroyuki Kimura, ${ }^{2}$ Yuma Sakamoto, ${ }^{2}$ Mamoru \\ Fukunaga, ${ }^{2}$ Yukio Noda, ${ }^{2}$ Masayasu Takeda, ${ }^{1}$ and Kazuhisa Kakurai ${ }^{1}$ \\ 1 Quantum Beam Science Directorate, Japan Atomic Energy Agency, Tokai, Ibaraki 319-1195, Japan \\ ${ }^{2}$ Institute of Multidisciplinary Research for Advanced Materials, Tohoku University, Sendai 980-8577, Japan
}

(Dated: August 23, 2018)

\begin{abstract}
We have performed simultaneous measurements of magnetic chirality by using polarized neutrons and electric polarization along the $b$-axis of single crystals of $\mathrm{YMn}^{4+}\left(\mathrm{Mn}_{1-x} \mathrm{Ga}_{x}\right)^{3+} \mathrm{O}_{5}$ with $x=0.047$ and 0.12 , in which nonmagnetic Ga-ions dilute $\mathrm{Mn}^{3+}$ spins. The $x=0.047$ sample exhibits high-temperature incommensurate (HT-ICM), commensurate (CM), and low-temperature incommensurate (LT-ICM) magnetic phases in order of decreasing temperature, whereas the $x=0.12$ sample exhibits only HT-ICM and LT-ICM phases. Here, the CM and LT-ICM phases are ferroelectric and weak-ferroelectric, respectively. Measurements conducted under zero field heating after various field-cooling conditions evidence that the microscopic mechanisms of the spin-driven ferroelectricity in the CM and LT-ICM phases are different: the magnetic chirality of $\mathrm{Mn}^{4+}$ cycloidal spins plays a dominant role in the LT-ICM phase, whereas the magnetic exchange striction by the $\mathrm{Mn}^{4+}-\mathrm{Mn}^{3+}$ chain plays a dominant role in the CM phase. The polarization of $\mathrm{YMn}_{2} \mathrm{O}_{5}$ flips upon CM to LT-ICM phase transition because the ferroelectricity driven by the magnetic chirality and the exchange striction provides opposite directions of polarization.
\end{abstract}

Many of the multiferroic compounds that have attracted much attention in recent years exhibit a giant magnetoelectric effect owing to the strong coupling between magnetism and ferroelectricity. The presence of this effect makes these multiferroic compounds potential candidates for industrial device applications. In addition, the coupling mechanism underlying the giant magnetoelectric effect is one of the central issues in this research field.

Multiferroic material $R \mathrm{Mn}_{2} \mathrm{O}_{5}$ (R: rare earth, Bi, and $\mathrm{Y})^{1}$ provides a unique opportunity for studying the coupling mechanism between magnetism and ferroelectricity. $R \mathrm{Mn}_{2} \mathrm{O}_{5}$, which has an orthorhombic structure with the Pbam space group, consists of edge-shared $\mathrm{Mn}^{4+} \mathrm{O}_{6}$ octahedral chain along the $c$-axis and pairs of $\mathrm{Mn}^{3+} \mathrm{O}_{5}$ pyramids connecting the $\mathrm{Mn}^{4+} \mathrm{O}_{6}$ octahedral chains. 2 Frustrated spin exchange interactions result in noncollinear magnetic structures and complex magnetic phases. $\frac{3}{2} R \mathrm{Mn}_{2} \mathrm{O}_{5}$ generally exhibits a magnetic phase transition from a paramagnetic to an incommensurate magnetic (HT-ICM) phase at $T_{N 1}$, which is typically $\sim 45 \mathrm{~K}$, followed by a successive magnetic phase transition into a commensurate $(\mathrm{CM})$ phase at $T_{C M}$, typically a few kelvins below $T_{N 1}$. Then, at lower temperature, the magnetic structure becomes incommensurate again (a phase referred to as LT-ICM) at $T_{N 2} \underline{\underline{4}, 5}$ These three magnetic phases exhibit different dielectric properties. The HT-ICM phase is paraelectric, whereas the CM phase exhibits spontaneous polarization concomitantly, and therefore, the $\mathrm{CM}$ phase is ferroelectric (FE). The LT-ICM phase is still ferroelectric, however the polarization is suppressed below the CM to LT-ICM phase transition temperature. Thus, the LT-ICM phase is recognized as weak ferroelectric (WFE). Remarkably, a rich variety of magnetoelectric effects have been identified in the LT-ICM phase of $R \mathrm{Mn}_{2} \mathrm{O}_{5}$. Such a variety of mag- netic and dielectric phases implies a complicated coupling mechanism in this system $\underline{\underline{6}}$

For the specific case of $\mathrm{YMn}_{2} \mathrm{O}_{5}, T_{N 1}=44 \mathrm{~K}, T_{C M}=$ $38 \mathrm{~K}$, and $T_{N 2}=20 \mathrm{~K}$. The magnetic modulation vectors for the HT-ICM, CM, and LT-ICM phases are $k=(0.254,0,0.484),(0.25,0,0.5)$, and $(0.292,0,0.48)$, respectively. The magnetic structure in the CM phase determined precisely by single crystal neutron diffraction is reported in Ref. 7. Characteristic is a spiral modulation of spins along the $c$-axis. The magnetic structure in the LT-ICM phase can be considered as a similar spiral with incommensurate modulation period from the polarized neutron diffraction. ${ }^{6}$ Spontaneous polarization along the $b$-axis appears concomitantly with the CM phase. Remarkably it is known that the polarization flips upon the CM to LT-ICM phase transition when the sample is poled at the CM phase. ${ }^{8-10}$

The exchange striction involving both $\mathrm{Mn}^{3+}$ and $\mathrm{Mn}^{4+}$ spins has been proposed for explaining magnetically driven ferroelectric polarization in $R \mathrm{Mn}_{2} \mathrm{O}_{5}$, based on powder magnetic structural analyses by using neutron diffraction. ${ }^{11,12}$ In this model, the polarization is proportional to the scalar product of neighboring spins, $\mathbf{P} \propto \mathbf{S}_{i} \cdot \mathbf{S}_{j}$. This model can reproduce the magnetic polarization in both CM and LT-ICM phases in $\mathrm{YMn}_{2} \mathrm{O}_{5}$

Noncollinear spin arrangements or cycloidal spin structures can break the inversion symmetry of the lattice through Dzyaloshinskii-Moriya antisymmetric interactions, which results in spontaneous polarization. ${ }^{13,14}$ In this case, the polarization is proportional to the vector product of neighboring spins, $\mathbf{P} \propto \mathbf{S}_{i} \times \mathbf{S}_{j}$. This relation has been confirmed in many multiferroic compounds such as orthorhombic $R \mathrm{MnO}_{3}, \mathrm{MnWO}_{4}, R \mathrm{Mn}_{2} \mathrm{O}_{5}$ and $\mathrm{Ni}_{2} \mathrm{~V}_{2} \mathrm{O}_{8}$ by using polarized neutron diffraction. $15-18$ Magnetic structural analyses using single-crystal neutron diffraction for various $R \mathrm{Mn}_{2} \mathrm{O}_{5}$ compounds confirmed 

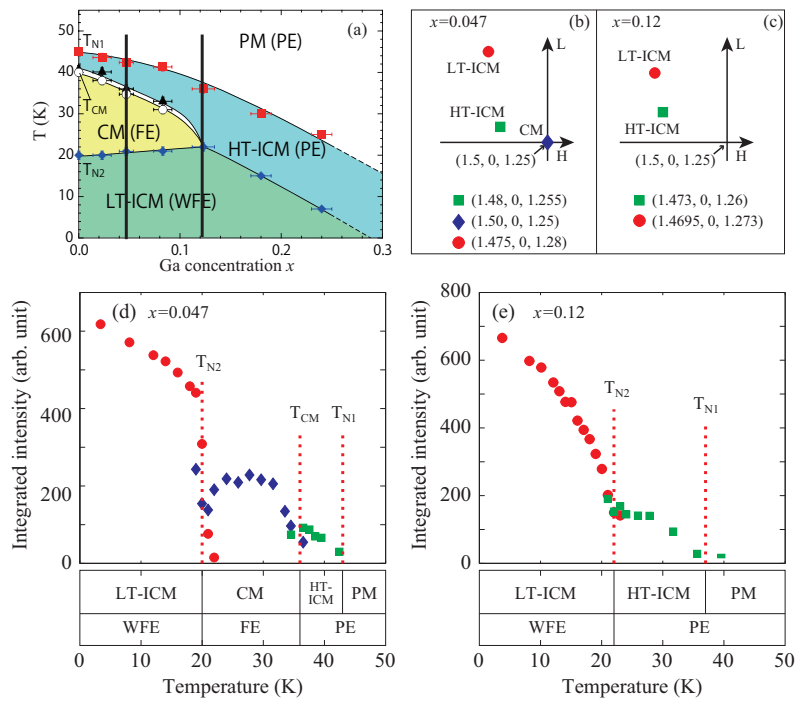

FIG. 1: (Color online) (a) Magnetic and dielectric phase diagram for $\mathrm{YMn}^{4+}\left(\mathrm{Mn}_{1-x} \mathrm{Ga}_{x}\right)^{3+} \mathrm{O}_{5}$ adopted from Ref. 22 . Vertical lines represent $x=0.047$ and 0.12 , which are used for the present study. (b) and (c) show CM and ICM peak geometry in the $(1.5,0,1.25)$ zone for $x=0.047$ and 0.12 , respectively. (d) and (e) show temperature dependence of magnetic peak intensities for $x=0.047$ and 0.12 , respectively, measured using unpolarized neutrons at the position specified in (b) and (c). Squares, diamonds, and circles represent data for HT-ICM, CM, and LT-ICM peaks, respectively.

the cycloidal spin structure of $\mathrm{Mn}^{4+}$ spins propagating along the $c$-axis $\frac{6.7,19-21}{21}$ This structure is also consistent with $b$-axis polarization.

Very recently, a magnetic and dielectric phase diagram was reported for $\mathrm{YMn}^{4+}\left(\mathrm{Mn}_{1-x} \mathrm{Ga}_{x}\right)^{3+} \mathrm{O}_{5}$ [Fig. 1(a)] wherein $\mathrm{Mn}^{3+}$ ions are selectively substituted by nonmagnetic $\mathrm{Ga}^{3+}$ ions $\stackrel{22}{2}$ The $\mathrm{Ga}^{3+}$ substitution does not affect the $\mathrm{Mn}^{4+}-\mathrm{Mn}^{4+}$ exchange interaction, and hence nor the cycloidal structure of $\mathrm{Mn}^{4+}$ spins, but it does affect the $\mathrm{Mn}^{4+}-\mathrm{Mn}^{3+}$ exchange interaction. Therefore, the $\mathrm{Ga}^{3+}$ substitution should dilute the contribution of the exchange striction mechanism to the spontaneous polarization. The phase diagram indicates that as Ga concentration increases, the $\mathrm{CM}(\mathrm{FE})$ phase is diminished below $x=0.12$. The LT-ICM (WFE) phase survives up to higher concentrations. This phase diagram suggests that ferroelectricity in the CM phase originates from the exchange striction, whereas weak ferroelectricity in the LT-ICM phase originates from the cycroidal structure of $\mathrm{Mn}^{4+}$ spins. A similar scenario has been hypothesized in Ref. 23 from dielectric measurements. So far, however, direct experimental evidence for this scenario is missing. In this Letter, we report results of simultaneous measurements of magnetic chirality and dielectric polarization in various cooling conditions and provide direct experimental evidence that the magnetic chirality of $\mathrm{Mn}^{4+}$ cycloidal spins plays a dominant role in the LTICM phase, whereas the magnetic exchange striction by the $\mathrm{Mn}^{4+}-\mathrm{Mn}^{3+}$ chain plays a dominant role in the $\mathrm{CM}$
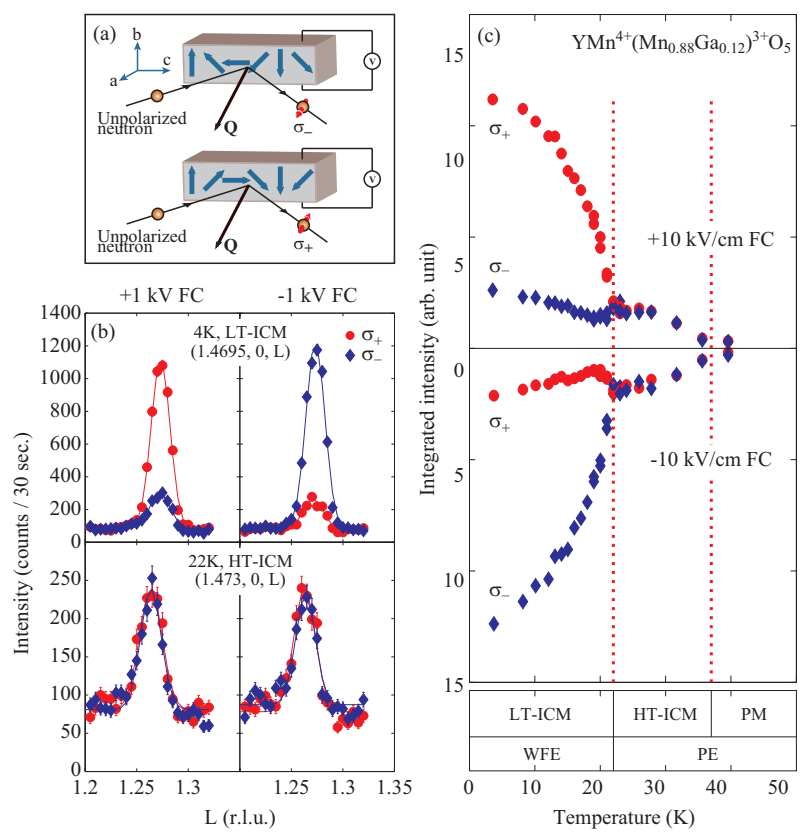

FIG. 2: (Color online) (a) Schematic drawings of the experimental geometry of polarized neutron diffraction. (b) $\sigma_{+}$ and $\sigma_{-}$profiles of LT-ICM peak (top) and HT-ICM (bottom) measured after cooling with electric fields of $+10 \mathrm{kV} / \mathrm{cm}$ (left) and $-10 \mathrm{kV} / \mathrm{cm}$ (right) for $x=0.12$. (c) Temperature dependence of $\sigma_{+}$(circles) and $\sigma_{-}$(diamonds).

phase.

We carried out simultaneous measurements of magnetic chirality by using polarized neutron diffraction and electric polarization for Ga-substituted $\mathrm{YMn}_{2} \mathrm{O}_{5}$ and studied the relation between chirality and polarization in each magnetic phase. We chose Ga concentrations of $x=0.047$ and 0.12 . These concentrations are specified by vertical bars in Fig. 1(a). Single crystals are grown in the same manner as those reported in Ref. 22. Magnetic phase transitions in these samples are verified using neutron diffraction, performed at the triple-axis spectrometer TAS-1 installed at the JRR-3 reactor, Tokai, Japan. Figures 1(d) and 1(e) show temperature dependence of magnetic peak intensity for both samples. The measured Q positions are schematically shown in Figs. 1(b) and 1 (c). The detailed change of the magnetic propagation vector has been reported in Ref. 22. Consistently with the phase diagram in Fig. 1(a), the $x=0.047$ sample exhibits HT-ICM, CM, and LT-ICM phases during cooling, whereas the $x=0.12$ sample has a single magnetic phase transition from HT-ICM to LT-ICM. Therefore, the former sample has both FE and WFE phases, and the latter sample has only the WFE phase.

Simultaneous measurements of polarized neutron diffraction and electric polarization were carried out also at TAS-1. The spin polarizations of the incident and diffracted neutrons, $\mathbf{P}_{\mathbf{i}}$ and $\mathbf{P}_{\mathbf{f}}$, are connected by the following formula when involving only magnetic scattering cross section from a magnetic structure: ${ }^{24} \mathbf{P}_{\mathbf{f}} \sigma=$ 


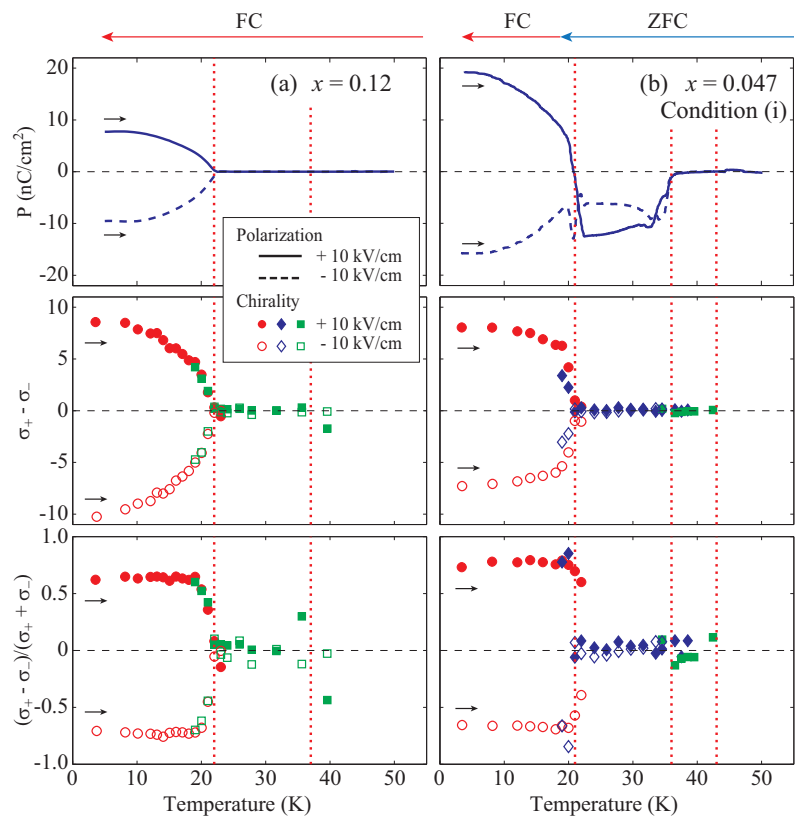

FIG. 3: (Color online) (a) Temperature dependence of electric polarization $P, \sigma_{+}-\sigma_{-}$as a measure of magnetic chirality, and normalized chirality $\left(\sigma_{+}-\sigma_{-}\right) /\left(\sigma_{+}+\sigma_{-}\right)$for (a) $x=0.12$ measured during the $\mathrm{ZFH}$ process after the $\mathrm{FC}$ process and (b) $x=0.047$ measured after ZFC down to $18 \mathrm{~K}$ followed by FC down to $4 \mathrm{~K}$.

$-\mathbf{P}_{\mathbf{i}}\left(\mathbf{M}^{*} \cdot \mathbf{M}\right)+\mathbf{M}^{*}\left(\mathbf{P}_{\mathbf{i}} \cdot \mathbf{M}\right)+\left(\mathbf{P}_{\mathbf{i}} \cdot \mathbf{M}^{*}\right) \mathbf{M}+i(\mathbf{M} \times$ $\left.\mathbf{M}^{*}\right)$, where $\mathbf{M}$ is a magnetic structure factor $\mathbf{M} \propto$ $\sum_{j} \mathbf{S}_{j \perp} e^{i \mathbf{Q} \cdot \mathbf{r}_{j}}$, and $\mathbf{M}^{*}$ is its complex conjugate. Here, $\sigma$, and $\mathbf{S}_{j \perp}$ are the total neutron cross section and a component of the $j-$ th spin perpendicular to the scattering vector $\mathbf{Q}$. The fourth term expresses the chiral structure contribution, which gives the final polarization parallel to $\mathbf{Q}$. We kept the incident neutrons unpolarized and analyzed the polarization of the diffracted neutrons using a Heusler analyzer with a spin flipper in front of the analyzer. A guide field around the sample was kept parallel to $\mathbf{Q}$ by a Helmholtz coil. Thus we analyze the final polarization $\mathbf{P}_{\mathbf{f}}$ parallel to $\mathbf{Q}$. Neutron cross sections with spin $(+), \sigma_{+}$, and $(-), \sigma_{-}$, can be measured with the spin flipper off (non-spin-flip channel) and the spin flipper on (spin-flip channel), respectively. From the guide field configuration, the polarizations of $\sigma_{+}$and $\sigma_{-}$are antiparallel and parallel to $\mathbf{Q}$, respectively. This is schematically shown in Fig. 2(a). Now Q-parallel component of $\mathbf{P}_{\mathbf{f}}$ is $\left(\sigma_{+}-\sigma_{-}\right) /\left(\sigma_{+}+\sigma_{-}\right)$, while total neutron cross section can be written as $\sigma=\sigma_{+}+\sigma_{-}$. In the present experimental setup, the previous formula can be simplified by extracting a Q-parallel component as: $\sigma_{+}-\sigma_{-}=i\left(\mathbf{M} \times \mathbf{M}^{*}\right)_{\mathbf{Q}}$. Therefore, the difference in cross sections, $\sigma_{+}-\sigma_{-}$, records the magnetic chirality $i\left(\mathbf{M} \times \mathbf{M}^{*}\right)$ directly.

First, let us discuss the simpler case shown in Fig. 1(e), that is, the phase transition from HT-ICM to LT-ICM for $x=0.12$. Figure 2 summarizes a typical data set

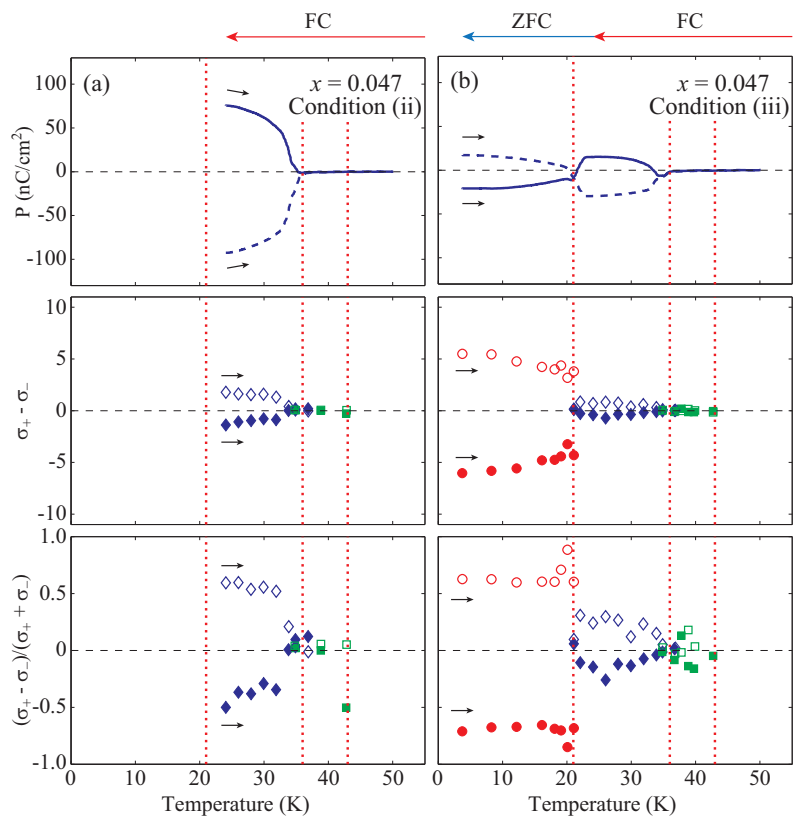

FIG. 4: (Color online) Analogous data plots to those of Fig. 3 for $x=0.047$ measured after (a) FC down to $24 \mathrm{~K}$, and (b) $\mathrm{FC}$ down to $24 \mathrm{~K}$ followed by ZFC down to $4 \mathrm{~K}$.

for the $x=0.12$ sample. The crystal was placed with $a$ and $c$-axes horizontal. Electrodes were attached so that an electric field can be applied along the $b$-axis. Figure 2(b) shows cross sections $\sigma_{+}$and $\sigma_{-}$measured at the $\mathbf{Q}$ positions specified in Fig. 1(c). Measurements were taken during a heating process without an electric field (zero-field heating, ZFH) after a cooling process with an applied electric field (field cooling, FC) of $10 \mathrm{kV} / \mathrm{cm}$ from 55 to $4 \mathrm{~K}$. At $22 \mathrm{~K}$ in the HT-ICM phase, which is paraelectric, there is no difference between $\sigma_{+}$and $\sigma_{-}$. This means that there is no magnetic chirality (or imbalance of chiral domains) in the paraelectric field. In contrast, at $4 \mathrm{~K}$ in the LT-ICM WFE phase, there is a difference between $\sigma_{+}$and $\sigma_{-}$. The magnitudes of these cross sections are reversed by changing the sign of the electric field. Therefore, the magnetic chirality flips when the electric polarization flips. We analyzed the peak profiles by fitting to a Gaussian function and derived the integrated intensity. The detailed temperature dependence of the integrated intensities of $\sigma_{+}$and $\sigma_{-}$is shown in Fig. 2(c). The difference only appears in the LT-ICM WFE phase.

The magnetic chirality, characterized as the difference $\sigma_{+}-\sigma_{-}$, is shown in Fig. 3(a), together with the electric polarization $P$ measured using pyrocurrent observed in a $\mathrm{ZFH}$ process. It is clearly shown that these two parameters follow identical temperature dependence in the LT-ICM phase. This behavior is exactly the same as that observed in $\mathrm{TbMnO}_{3}{ }^{15}$ In Fig. 3(a), we show the normalized chirality $\left(\sigma_{+}-\sigma_{-}\right) /\left(\sigma_{+}+\sigma_{-}\right)$in the bottom panel. This parameter remains nearly constant up to $T_{N 2}$ at $\sim 0.6$. Note that the normalized chirality should 
be less than 1 even when the magnetic structure has a single chiral domain in case of $\mathrm{YMn}_{2} \mathrm{O}_{5}$ since $\mathrm{Mn}$ spins form a cycloidal structure with elongated magnetic moment along the $a$-axis,,$\underline{7}$ and thence $\mathbf{S}_{j \perp}$ forms an ellipsoid at $\mathbf{Q}$ we measure. (This fact corresponds to appreciable $\sigma_{-}\left(\right.$or $\left.\sigma_{+}\right)$of the $+10 \mathrm{kV} / \mathrm{cm}$ (or $-10 \mathrm{kV} / \mathrm{cm}$ ) data at $4 \mathrm{~K}$ in Fig. 2(b).) We calculated the normalized chirality at $(1.5,0,1.25)$ using the magnetic structure of the $\mathrm{CM}$ phase in $\mathrm{YMn}_{2} \mathrm{O}_{5}$ reported in Ref. 7 with single chirality. Calculated value is 0.52 which is reasonably consistent with the observed value 0.60 . This means that, through the FC process, the sample was fully poled and contained a single magnetic domain. It should be noted here that, in the LT-ICM phase of this sample, polarization and chirality have the same sign.

As a second case, we made similar measurements for the $x=0.047$ sample. Because this sample has two dielectric phases, FE and WFE, we employed three different FC conditions: (i) zero-field cooling (ZFC) from 55 to $18 \mathrm{~K}$, followed by $\mathrm{FC}$ from 18 to $4 \mathrm{~K}$, (ii) FC from 55 to $24 \mathrm{~K}$, and (iii) $\mathrm{FC}$ from 55 to $24 \mathrm{~K}$, followed by ZFC from 24 to $4 \mathrm{~K}$. Figure $3(\mathrm{~b})$ shows the plots of polarization $P$, chirality $\left(\sigma_{+}-\sigma_{-}\right)$, and normalized chirality $\left(\sigma_{+}-\sigma_{-}\right) /\left(\sigma_{+}+\sigma_{-}\right)$for condition (i). This condition corresponds to poling of the LT-ICM WFE phase only. In this case, $P$ and the chirality in the LT-ICM phase have the same sign, and they are reversible by changing the electric field. Thus, the behavior in the LT-ICM phase is consistent with that in the $x=0.12$ sample. In the $\mathrm{ZFH}$ process, there remains a finite polarization in the CM phase, likely resulting from an incipient imbalance of ferroelectric domains; nevertheless, the chirality disappears. This shows that the ferroelectricity in the CM phase does not require the magnetic chirality.

In contrast, when the sample is poled in the CM-FE phase [condition (ii), Fig. 4(a)], both $P$ and the chirality appear in the $\mathrm{CM}$ phase. Here $P$ in the $\mathrm{CM}$ phase is about four times larger than that in the LT-ICM phase in Fig. 3(b). (Note the difference in the vertical scales between Figs. 3 and 4.) $P$ and the chirality are again reversible by changing the electric field as a normal character of ferroelectricity; however, they have opposite signs: for example, $P$ is positive with $+10 \mathrm{kV} / \mathrm{cm}$ but the chirality is negative.

In both conditions (i) and (ii) [Fig. 3(b) and 4(a)], the normalized chirality reaches $\sim 0.6$; that is, both poled CM and LT-ICM phases have a single magnetic domain but opposite chirality. This means that nearly the same magnetic structure gives opposite spontaneous polarization in the CM and LT-ICM phases. This is clearly shown in Fig. 4(b), which shows the data in condition (iii). The sample is once poled in the CM phase, then zero-fieldcooled to $4 \mathrm{~K}$. In this case, the magnetic structure poled in the $\mathrm{CM}$ phase is preserved down to $4 \mathrm{~K}$, and therefore, the system gives nearly identical values of $P$ and normalized chirality in the opposite direction to that of the condition (i) [Fig. 3(b)] in the LT-ICM phase. Thus, the chiral domain poled in the CM phase is preserved in

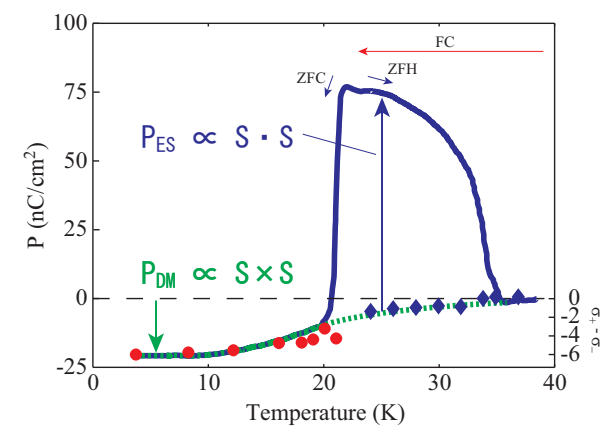

FIG. 5: (Color online) Total polarization (solid line) together with the magnetic chirality in $\mathrm{YMn}_{2} \mathrm{O}_{5}$ when the system is poled at the CM phase. Diamonds and circles are plotted $\sigma_{+}-\sigma_{-}$of the CM phase in Fig. 4(a) and those of LT-ICM in Fig. 4(b), respectively. $P_{D M}$ contribution is shown by a dotted line.

the LT-ICM phase with the ZFC process. In contrast, in condition (i) in Fig.3(b), the chirality vanishes at $T_{N 2}$. This indicates that the chiral domain poled in the LTICM phase is not preserved in the CM phase even with the ZFH process. This is also consistent with the observation in Fig. 4(b) showing that the chirality vanishes at $T_{N 2}$ in the ZFH process. These facts indicate that the ferroelectricity in the CM and LT-ICM phases has different origins although it arises from nearly identical magnetic structures. Moreover, the results in Fig. 3 strongly support that magnetic chirality is the origin of weak ferroelectricity in the LT-ICM phase.

Based on the above, the total electric polarization in $\mathrm{YMn}_{2} \mathrm{O}_{5}$ can be expressed as a summation of two components: $P=P_{E S}+P_{D M}$, where $P_{E S}\left(\propto \mathbf{S}_{\mathbf{i}} \cdot \mathbf{S}_{\mathbf{j}}\right)$ is dominant in the CM phase and $P_{D M}\left(\propto \mathbf{S}_{\mathbf{i}} \times \mathbf{S}_{\mathbf{j}}\right)$ is dominant in the LT-ICM phase. This is schematically shown in Fig. 5. When the system is poled in the CM phase, it exhibits a single magnetic domain and $P_{E S}$ has positive polarization. However, this magnetic structure possesses a chirality that gives negative $P_{D M}$. Upon ZFC across the CM to the LT-ICM phase transition, $\mathbf{S}_{\mathbf{i}} \cdot \mathbf{S}_{\mathbf{j}}$ information is lost by the incommensurate modulation period while $\mathbf{S}_{\mathbf{i}} \times \mathbf{S}_{\mathbf{j}}$ information is preserved. Therefore, only $P_{D M}$ survives and the electric polarization flips. This model is also evidenced by that $P$ in the $\mathrm{CM}$ phase in Fig. 4(b) is much smaller than Fig. 4(a). In case of Fig. 4(b), the sample once enters the LT-ICM phase after poled at the CM phase, and bulk ferroelectricity by $\mathbf{S}_{\mathbf{i}} \cdot \mathbf{S}_{\mathbf{j}}$ is diminished by the incommensurate structure of LT-ICM. Thus, the ferroelectricity does not fully recover when the system reenter the CM phase on ZFH.

In summary, we performed simultaneous measurements of magnetic chirality and electric polarization for $\mathrm{YMn}^{4+}\left(\mathrm{Mn}_{1-x} \mathrm{Ga}_{x}\right)^{3+} \mathrm{O}_{5}$ in which nonmagnetic $\mathrm{Ga}^{3+}$ dilutes the contribution of $\mathbf{S}_{\mathbf{i}} \cdot \mathbf{S}_{\mathbf{j}}$ to the ferroelectricity. Polarization, magnetic chirality, and normalized magnetic chirality measured under various field-cooling conditions evidence that the ferroelectric polarizations in the 
CM and LT-ICM phases are dominated by components $P_{E S}\left(\propto \mathbf{S}_{\mathbf{i}} \cdot \mathbf{S}_{\mathbf{j}}\right)$ and $P_{D M}\left(\propto \mathbf{S}_{\mathbf{i}} \times \mathbf{S}_{\mathbf{j}}\right)$, respectively. Owing to the similar magnetic and dielectric properties of $R \mathrm{Mn}_{2} \mathrm{O}_{5}$, this phenomenon is likely to be valid for the most family compounds of this system. The signs of $P_{E S}$ and $P_{D M}$ may depend on compounds with different $R$ ions. In the specific case of $R=\mathrm{Y}$, the polarization flip upon the CM to LT-ICM phase transition can be accounted for by the different signs of $P_{E S}$ and $P_{D M}$.

This work is supported by Grant-In-Aid for Scientific Research (B) No. 16340096, and Scientific Research (A) No. 21244051 and Grant-In-Aid for Scientific Research on Priority Areas "Novel States of Matter Induced by Frustration" No. 19052001.
1 N. Hur, S. Park, P. A. Sharma, J. S. Ahn, S. Guha, and S.-W. Cheong, Nature 429, 392 (2004).

2 J. A. Alonso, M. T. Casais, M. J. Martínez-Lope, J. L. Martínez, and M. T. Fernández-Díaz, J. Phys.: Condens. Matter 9, 8515 (1997).

${ }^{3}$ L. C. Chapon, G. R. Blake, M. J. Gutmann, S. Park, N. Hur, P. G. Radaelli, and S.-W. Cheong, Phys. Rev. Lett. 93, 177402 (2004).

${ }^{4}$ I. Kagomiya, H. Kimura, Y. Noda, and K. Kohn, J. Phys. Soc. Jpn. 70, S145 (2001).

5 Y. Noda, H. Kimura, M. Fukunaga, S. Kobayashi, I. Kagomiya, and K. Kohn, J. Phys.: Condens. Matter 20, 434206 (2008).

6 J.-H. Kim, S.-H. Lee, S. I. Park, M. Kenzelmann, A. B. Harris, J. Schefer, J.-H. Chung, C. F. Majkrzak, M. Takeda, S. Wakimoto, S. Y. Park, S.-W. Cheong, M. Matsuda, H. Kimura, Y. Noda, and K. Kakurai, Phys. Rev. B 78, 245115 (2008).

7 H. Kimura, S. Kobayashi, Y. Fukuda, T. Osawa, Y. Kamada, Y. Noda, I. Kagomiya, and K. Kohn, J. Phys. Soc. Jpn. 76, 074706 (2007).

8 A. Inomata and K. Kohn, J. Phys.: Condens. Matter 8, 2673 (1996).

9 I. Kagomiya, S. Matsumoto, K. Kohn, Y. Fukuda, T. Shoubu, H. Kimura, Y. Noda, and N. Ikeda, Ferroelectrics 286, 167 (2003).

10 R. P. Chaudhury, C. R. D. Cruz, B. Lorenz, Y. Y. Sun, C. W. Chu, S. Park, and S.-W. Cheong, J. Phys.: Conference Series 150, 042013 (2009).

11 L. C. Chapon, P. G. Radaelli, G. R. Blake, S. Park, and S.-W. Cheong, Phys. Rev. Lett. 96, 097601 (2006).

12 G. R. Blake, L. C. Chapon, P. G. Radaelli, S. Park, N. Hur, S.-W. Cheong, and J. Rodríguez-Carvajal, Phys. Rev. B 71, 214402 (2005).
13 H. Katsura, N. Nagaosa, and A. V. Balatsky, Phys. Rev. Lett. 95, 057205 (2005).

14 I. A. Sergienko and E. Dagotto, Phys. Rev. B 73, 094434 (2006).

15 Y. Yamasaki, H. Sagayama, T. Goto, M. Matsuura, K. Hirota, T. Arima, and Y. Tokura, Phys. Rev. Lett. 98, 147204 (2007).

16 H. Sagayama, K. Taniguchi, N. Abe, T.-h. Arima, M. Soda, M. Matsuura, and K. Hirota, Phys. Rev. B 77, 220407 (2008).

17 M. Fukunaga, Y. Sakamoto, H. Kimura, Y. Noda, N. Abe, K. Taniguchi, T. Arima, S. Wakimoto, M. Takeda, K. Kakurai, and K. Kohn, Phys. Rev. Lett. 103, 077204 (2009).

18 I. Cabrera, M. Kenzelmann, G. Lawes, Y. Chen, W. C. Chen, R. Erwin, T. R. Gentile, J. B. Leão, J. W. Lynn, N. Rogado, R. J. Cava, and C. Broholm, Phys. Rev. Lett. 103, 087201 (2009).

19 Y. Noda, H. Kimura, Y. Kamada, T. Osawa, Y. Fukuda, Y. Ishikawa, S. Kobayashi, Y. Wakabayashi, H. Sawa, N. Ikeda, et al., Physica B: Condensed Matter 385-386, 119 (2006).

20 C. Vecchini, L. C. Chapon, P. J. Brown, T. Chatterji, S. Park, S.-W. Cheong, and P. G. Radaelli, Phys. Rev. B 77, 134434 (2008).

21 P. G. Radaelli, C. Vecchini, L. C. Chapon, P. J. Brown, S. Park, and S.-W. Cheong, Phys. Rev. B 79, 020404 (2009).

22 H. Kimura, Y. Sakamoto, M. Fukunaga, H. Hiraka, and Y. Noda, Phys. Rev. B 87, 104414 (2013).

23 M. Fukunaga and Y. Noda, J. Phys. Soc. Jpn. 79, 054705 (2010).

24 M. Blume, Phys. Rev. 130, 1670 (1963). 